\title{
Lo social en el futuro de la economía aplicada: Una aportación sociocibernética
}

\section{Chaime MARCuELlo SeRVós}

Universidad de Zaragoza, Facultad de Ciencias Sociales y del Trabajo, c/ Violante de Hungría, 3, 50009 Zaragoza, España. E-mail: chaime@unizar.es

\section{RESUMEN}

Partiendo de una perspectiva sociocibernética se proponen algunos interrogantes para pensar lo social en el futuro de la economía aplicada. Primero, se aborda lo social y lo económico considerando el debate del 'embeddedness'. Segundo, se plantea la tensión de las ciencias sociales entre describir y prescribir atendiendo a la gran transformación provocada por la digitalización de los procesos sociales. Tercero, se discute la influencia de los reguladores de los mercados financieros, donde el 'oráculo' de Draghi en agosto de 2017 sirve como ejemplo. Por último, se platean unas claves para pensar lo futuro.

Palabras clave: 'Embeddedness', futuro, apertura, digitalización.

\section{The Social in the Future of the Applied Economy: A Sociocybernetic Contribution}

\begin{abstract}
Starting from a socio-cybernetic perspective, some questions are proposed to think about the social future of applied economics. First, it addresses the social and the economic considering the debate of 'embeddedness'. Second, there is the tension of the social sciences between describing and prescribing on the framework of the great transformation provoked by the digitization of social processes. Third, the influence of financial market regulators is discussed, where Draghi's 'oracle' in August 2017 serves as an example. Finally, some keys are posited to think about the future. Keywords: Embeddedness, Future, Openness, Digitalization.
\end{abstract}

Clasificación JEL: B20, G41, Z13

Artículo recibido en octubre de 2017 y aceptado en noviembre de 2017

Artículo disponible en versión electrónica en la página www.revista-eea.net, ref. ə-36104 
"Echo de menos el futuro. Hoy en día nuestras expectativas son demasiado bajas"

(Lanier, 2014, 421)

El futuro debería ser nuestro teatro,

(Lanier 2014, 422)

\section{INTRODUCCIÓN}

Los sistemas sociales tienden a la simplificación de rutinas y hábitos. Como sujetos reiteramos conductas buscando seguridades y encajando la interpretación del mundo que nos rodea dentro de los códigos socialmente compartidos. Nos sujetamos a esas cosas que damos por buenas por mor de repeticiones construidas con el paso del tiempo. Son capas de palabras, de lenguaje, unas resultado de significados sedimentados e instituidos socialmente; otras consecuencia de las regularidades descritas y observadas en el mundo físico.

Por eso esperamos que mañana salga el sol por el este y, como siempre, se esconda por el oeste. Esperamos que ese orden y esa lógica del mundo se mantengan estables, porque bastante inestabilidad nos produce nuestra condición humana, frágil, contingente y mortal. Experimentamos el cambio en nuestro propio cuerpo y en el entorno más de lo que nos gustaría. Buscamos 'la' racionalidad en el mundo y construimos 'una' racionalidad ad hoc con la que conseguir seguridad y estabilidad en nuestra percepción de los fenómenos y en la comprensión de los mismos. Otra cosa es que con ello no están dadas de suyo ni seguridad, ni estabilidad, ni certidumbre pues sólo son estrategias conservadoras de deseos, de propiedades o de jerarquías. De hecho, el tipo de simplificación que producen los sistemas sociales es, primero, cognitivo, después, cultural y, por último, material. No necesariamente en este orden.

Cada individuo, cada generación vive una sola vez, asimilando, reinterpretando y reestructurando los códigos socialmente disponibles, legados por sus antecesores y filtrados para la siguiente posteridad. Cada sujeto cuenta con un núcleo básico de verdades apodícticas, más o menos conscientes, desde las cuales interpreta y entiende cómo se mueve su cosmos. Es imposible salirse de los límites de ese cosmos si no se acepta que existe la posibilidad de otra perspectiva. Y ya sabemos, desde hace muchos siglos, que es muy complicado cambiar de mirada y salir de la caverna.

De ese modo, el futuro se presenta como proyección en el tiempo de los sucesos aprehendidos previamente. Se acepta como regla básica que las causas de las cosas son las que son, las que se ven. Partiendo de esa regularidad sólo es necesario comprender las reglas, las leyes del mundo para saber cómo serán mañana las cosas. Esta concepción se corresponde con un modelo trivial -input 
output- que suele entrar en contradicción con el funcionamiento no trivial de lo social. Nos quedamos en el mundo que nuestro lenguaje nos muestra, por eso no somos capaces de percibir lo que no percibimos. Es decir, mientras no se da un salto de reflexividad, un descentramiento de la perspectiva, sólo se ve lo que se ve.

Teniendo en cuenta estas coordenadas iniciales, aquí intentaremos plantear algunos interrogantes para pensar lo social en el futuro de la economía aplicada. Retomaremos sucintamente el problema del embeddedness (Granoveter, 1985), después la gran transformación provocada por la digitalización de los procesos sociales (Lanier, 2014) y la influencia social de los reguladores de los mercados financieros, donde el 'oráculo' de Draghi en agosto de 2017 sirve como ejemplo. Por último, propondremos unas claves para pensar lo futuro.

\section{LO SOCIAL Y LO ECONÓMICO}

En sentido estricto, sólo merece la pena una versión aplicada de la economía que sea capaz de resolver problemas prácticos, tal y como se puede decir de la medicina o de cualquier campo del conocimiento. La etimología del término nos recuerda que estamos ante un saber dónde hay dos dimensiones oikos -casa, hogar, unidad social donde se atendía a las necesidades y se compartían alimentos- y nomos -ley-. El objeto propio de la economía está incrustado (embedded) directamente en la vida cotidiana, tanto si se considera el estudio de los mercados, las formas satisfacción de las necesidades humanas como si se centra en la toma de decisiones.

Cabría decir que la economía es un quehacer entre los deseos y la realidad, entre la despensa y el banquete, deudor de la abundancia siempre deseada y de la escasez consustancial a los límites de la naturaleza. Como si fuera una disciplina hija de Poros y Penía que hereda la tensión entre riqueza y pobreza, una tensión dinámica donde maximizar los recursos en el juego de la oferta y la demanda. Es una ciencia social que necesariamente ha producido, produce y producirá teorías e ideas más o menos exitosas de los fenómenos en cuestión.

En más de una ocasión se ironiza enfatizando que los economistas siempre aciertan cuando explican a posteriori, una vez sucedidos los fenómenos; el gran reto es adelantar escenarios buscando explicaciones acertadas que permitan comprender mejor el funcionamiento del sistema social, pero también la capacidad para intervenir, pronosticar y anticipar. Algo donde la falta de acierto y la incapacidad para predecir -por ejemplo, la crisis de las subprime y su impacto internacional- ha dejado un estigma difícil de superar.

La dificultad radica en la irreversibilidad de los fenómenos y en la multiplicidad de variables. Como propuso Heinz Von Foerster's en su teorema número dos "The hard sciences are successful because they deal with the soft problems; the soft sciences are struggling because they deal with the hard 
problems" (Foerster, 2003, 191). Y este mismo autor recordaba que: "the scientist who works in the soft sciences is faced with a formidable problem: he cannot afford to loose sight of the full complexity of his system, on the other hand it becomes more and more urgent that his problems be solved" (Foerster, 2003, 192). Los problemas son innumerables dada la dinámica de la vida humana.

Desde otra perspectiva, Karl Polanyi tenía claro que "por lo general las relaciones sociales de los hombres engloban su economía" (Polanyi, 1989, 76). Y en esa misma línea Granoveter (1985) planteó su ya clásico trabajo "Economic Action and Social Structure: The Problem of Embeddedness" que comenzaba recordando que la teoría sociológica está preocupada por responder a cómo afectan las relaciones sociales al comportamiento y a las instituciones, para terminar diciendo: "I believe the embeddedness argument to have very general applicability and to demonstrate not only that there is a place for sociologists in the study of economic life but that their perspective is urgently required there" (Granoveter, 1985, 507).

Así, aunque se quiera postular lo social y lo económico como dos universos simbólicos separados resultado de la modernización de la sociedad y de los mercados, el contraejemplo que facilita la crisis sistémica de 2008 muestra la interconexión de ambas dimensiones. Cuanto más se intenta explicar lo sucedido, más evidentes son las interrelaciones entre las decisiones individuales en un contexto político y jurídico que permitía un modo de gestión financiera alejado de los mecanismos de control que podrían haber intervenido de manera contra-cíclica. Las expectativas de unos y otros estuvieron atravesadas por elementos emocionales, morales y conductuales alejados de los supuestos de una racionalidad económica 'normalizada'. Pese a ello, los efectos fueron menores que la gran crisis de 1929 (Galbraith, 2008) porque en su momento se incorporaron al sistema mecanismos de protección donde compartir riesgos.

No obstante, lo económico puede ser interpretado como algo con su propia dinámica interna, con su racionalidad. Pueden construirse modelos, agregarse datos y definir variables que permitan ofrecer imágenes que explican momentos puntuales. Pero el objeto de la economía aplicada siempre se rebosa por su carácter dinámico. Además, lo social y lo económico son procesos donde las emociones juegan un papel central, más que la simple suma de estadísticas (Scheff, 1990), (Bericat, 2012). La mera formulación de expectativas o la introducción de ruido, de rumores en el sistema económico tienen efectos directos en su equilibrio. Si en el sistema social se identifican los mecanismos de feedback como de feedforward cabe la posibilidad de adaptarse al entorno en función de las decisiones previamente trazadas. Nada garantiza tener el control absoluto del sistema pero sí, al menos, elementos de reflexividad que permiten respuestas adaptativas (Amozurrutia, 2012). 
Si nos fijamos en el desarrollo actual, contamos con disciplinas y subdisciplinas separadas entre sí. Cada una atiende su rincón especializado, distinguiendo su objeto y método, para demarcar su área de conocimiento, creando corpus teóricos particulares que se corresponden con trozos de la realidad donde se investiga empíricamente. Pero esto no sirve. Parafraseando a Keneth E. Boulding, cabe afirmar que para dedicarse a cualquier asunto relacionado con el conocimiento, especialmente de la realidad social, es necesario un enfoque holístico e integrador. Es decir, se requiere de análisis y perspectivas que vayan más allá de la división del conocimiento en áreas cerradas y sin comunicación, intentado ampliar la propia perspectiva.

Boulding lo describía así: "para investigar un segmento concreto del mundo real, como es el movimiento sindical, hay que hacerlo no sólo con las herramientas del economista, sino del sociólogo, del politólogo, del antropólogo e incluso del filósofo y del teólogo. Llegué a la conclusión de que todas las ciencias sociales estudian lo mismo -el sistema social-, pero desde distintos puntos de vista, y empecé a interesarme por la integración de las ciencias sociales, que finalmente me llevaría a ser uno de los padres fundadores de la actual International Society for the Systems Sciences, y por la unidad del conocimiento humano, preocupación que ya no me ha abandonado jamás” (Boulding, 1994, 86).

Esa convicción es algo a recuperar, especialmente cuando se piensa lo social en el futuro de la economía aplicada. Los problemas a los que se ha de responder requieren cada vez más de esa mirada integradora que busca comprender los retos sociales de nuestro tiempo. Un mundo radicalmente transformado por la revolución microelectrónica.

\section{PRESCRIBIR Y DESCRIBIR}

Todavía no tenemos suficiente distancia para comprender la gran transformación provocada con la incorporación de las tecnologías de la información y de la comunicación (TIC) a la vida cotidiana. Se han escrito numerosas obras mostrando cambios e innovaciones, incluso dibujando el salto de etapa evolutiva para el conjunto de la Humanidad (Marcuello, 2010; Aramburu y Marcuello-Servós, 2017). Tenemos conciencia de estar en un plano distinto, equivalente al tránsito del neolítico o al de la revolución industrial (Lanier, 2014). Este cambio afecta a las estructuras básicas de la vida social en su conjunto. Es algo cada vez más evidente en las formas de interacción tanto entre individuos como en las organizaciones.

Después de La gran transformación de Karl Polanyi (1989) [1944] donde se mostraba los efectos del apogeo y el declive del homo economicus, quedaron desnudos los pilares de la economía y de la sociedad de aquella época. Polanyi supo analizar el desenlace político y las nefastas consecuencias en la génesis de 
los totalitarismos del siglo XX. En esa obra se mostraban los efectos de la economía de mercado en la sociedad de mercado que se convertía en subalterna frente a cualquier otra prioridad. Se había dado la vuelta, se había transmutado el orden de valores.

Polanyi (1989) mostró las consecuencias al hacer que prevalezcan los argumentos económicos. E insistía en desnudar el modelo de mercado, diciendo que "en la medida en que está íntimamente unido a un móvil particular que le es propio -el pago en especie o el trueque-, es capaz de crear una institución específica, más precisamente, es capaz de crear el mercado. A fin de cuentas, ésta es la razón por la que el control del sistema económico por el mercado tiene irresistibles efectos en la organización de la sociedad en su conjunto: esto significa simplemente que la sociedad es gestionada en tanto que auxiliar al mercado. En lugar de que la economía se vea marcada por las relaciones sociales, son las relaciones sociales quienes se ven encasilladas en el interior del sistema económico. La importancia vital del factor económico para la existencia de la sociedad excluye cualquier otro tipo de relación, pues, una vez que el sistema económico se organiza en instituciones separadas, fundadas sobre móviles determinados y dotadas de un estatuto especial, la sociedad se ve obligada a adoptar una determinada forma que permita funcionar a ese sistema siguiendo sus propias leyes. Es justamente en ese sentido en el que debe ser entendida la conocida afirmación de que una economía de mercado únicamente puede funcionar en una sociedad de mercado" (Polanyi 1989,105). En ese contexto, lo económico marcaba los límites de lo social.

Cualquier mirada hacia el futuro dibujaba un horizonte donde se creía que la desregulación y el libre juego del mercado autorregulado funcionarían por sí mismos. Pese a las contradicciones posteriores, para muchos sigue siendo la mejor opción: la libertad de cada individuo por encima de cualquier otra condición. Algo que ciertamente suena bien, si se cuenta con los recursos y la salud suficiente como para no necesitar de nadie más. Como contrapunto, la invención del estado social y democrático de derecho ha permitido vivir en un modelo complementario, al menos en el seno de la Unión Europea, que ha facilitado unos niveles de desarrollo social impensables al finalizar la segunda guerra mundial (Sotelo, 2010).

Con el cambio de contexto tecnológico, las condiciones de posibilidad de la vida cotidiana -de lo social en sí mismo- se han transformado y sigue todavía en proceso. Una parte de las formas de interacción social se han digitalizado. De tal modo que para algunos autores estamos ya en el albor de un cambio de la naturaleza humana como postulan de manera fuerte desde el movimiento transhumanista (Bostrom, 2001) o, de modo más blando, con la idea de Homo Zapiens "is not only a generation doing things differently from previous generations, Homo Zappiens is an exponent of societal changes related to globalization, 
individualization and the increasing uses of technology in our lives" (Veen and Vrakking, 2006, 5).

Esto nos enfrenta a dos tareas clásicas en las ciencias sociales, en general, y en la sociología, si es que se puede decir en singular. Una es la de describir el sistema social en el que vivimos; otra prescribir y proponer horizontes a donde orientar el rumbo, el futuro de la sociedad. Desde la perspectiva sociocibernética está claro que en hay algunas cosas que no suceden por casualidad y, por tanto, podemos trazar rumbos (Marcuello, 2006).

Para Veen and Vrakking se da una condición clave: "Future society demands that its citizens be able to cope with this complexity both in their private and working lives. Social networks of individuals have grown and have become more complex than before, and so have economic networks that have evolved at a global scale resulting in multinational or global economies" (Veen and Vrakking, 2006, 13). Algo que enlaza con la pregunta de Jaron Lanier sobre ¿Quién controla el futuro? Las TIC están transformando el mundo en el que nos movemos y las formas con las que interaccionamos.

Lanier (2014) propone una economía digital distinta, un entorno regulado de tal modo que se fortalezcan las clases medias como soporte general del sistema social. Y lo denomina "economía humanista en red". Es un modelo de economía aplicada que postula de modo prescriptivo: "A medida que la tecnología avanza, el estudio de la economía debe ser menos abstracto. Tradicionalmente, la disciplina de la economía se ha basado en los patrones de resultados que surgían de las reglas que influían sobre el comportamiento social de los seres humanos. Se centraba en estudiar cómo afectaban las políticas a las situaciones resultantes. Pero, con cada año que pasa, la economía debe interesarse más por el diseño de las máquinas que actúan como intermediarias del comportamiento social. Un sistema de información en red influye sobre la conducta de las personas de una manera más directa, detallada y literal que las políticas. Otra forma de expresarlo es decir que el estudio de la economía debe convertirse en una versión sistémica a gran escala del diseño de interfaces de usuario" (Lanier, 2014, 416)

¿Cómo afectan las TIC a los procesos sociales? ¿Qué pasa con lo social en un universo cada vez más mediado por computadoras y artilugios electrónicos? ¿Cómo influye en el análisis de los procesos económicos? Las respuestas están abiertas. En esto cabe esperar quietos o dar pasos explorando o bien caminar hacia un norte, con un rumbo preestablecido. Las TIC han modificado ya la percepción y la vivencia tanto de la distancia como del tiempo: han modificado lo social y lo económico. Y han multiplicado la información disponible, aunque no es tan obvio que ésta se haya digerido en forma de conocimiento socialmente distribuido. 
Las formas de interacción social se han multiplicado por medio de la redes sociales. Las conversaciones adquieren formatos no presenciales, se habla en la red, escribiendo tweets o mensajes inconexos, compartiendo fotos y videos que desbordan la capacidad individual de procesar los flujos de datos y la información existente. Mientras, el dinero se volatiliza y se digitaliza. La hiperconectividad produce cansancio (Han, 2012). Se comparten asuntos que potencian modelos de interacción basados más en la confianza (ciega) que en la crítica sustentada en datos, como si hubiéramos olvidado la encomienda kantiana del sapere aude.

\section{EL ORÁCULO DE DRAGHI}

Los efectos de esta gran transformación se perciben en la economía global. Una sociedad post-capitalista más ubicua y líquida (Drucker, 1993), donde se observa lo que cabe denominar dimensión mística y mistificadora de actores centrales en el sistema. Los datos, las cifras y las magnitudes son utilizadas por los responsables de la supervisión de los mercados financieros como formas de evangelizar. Un ejemplo es la intervención de Mario Draghi en el Economic Policy Symposium de Jackson Hole en agosto de 2017.

Parte de una idea de 'consenso social' para defender el mercado abierto como propuesta de fe en la que creer y a la que apoyar porque está siendo debilitada. Constata emociones, entre ellas, la sombra del miedo: "This is because fears about fairness, safety and equity ultimately reflect a lack of trust in other countries' regulation and enforcement” (Draghi, 2017, §9). Y recuerda como eje central de su argumento que "when thinking about the global economy, one of the key ingredients for raising productivity is openness. Open trade, investment and financial flows play a key role in the diffusion of new technologies across borders that drive forward efficiency improvements" (Draghi, 2017, §4).

Draghi habló en un presente que quiere un determinado futuro. Tiene claro que sus palabras van a volar por las redes, amplificando su contenido en forma de eco con impacto directo en los flujos de dinero y las finanzas en el mundo. No tiene problema en usar valores intangibles para propones conclusiones operativas: "Multilateral cooperation is crucial in responding to concerns about fairness, safety and also equity” (Draghi, 2017, §56). Reclama la convergencia normativa para proteger a la gente de las consecuencias de la apertura de los mercados y lo hace desde un diseño futuro, de una manera de entender la economía aplicada a lo social. Habla para defender un modelo, el de la Unión Europea, el de su banco, que pese a las grandes contradicciones que arrastra es de lo mejor que se puede encontrar en el planeta. Tras el discurso de Draghi en Jackson Hole el euro incrementó en 1,3\% su valor frente al dólar (Badkar, 2017), es una correlación con visos de causalidad implícita, ampliada y 
multiplicada por flujos de bits, gracias a las TIC. Sin ellas, el eco de esa reunión en Wyoming, no habría tenido lugar.

\section{CLAVES PARA EL FUTURO}

La investigación e incorporación de lo subjetivo, de lo intangible y de lo emocional en las ciencias sociales está inextricablemente conectado con el factor humano (Moreno-Jiménez, 2006; García Lizana \& Moreno-Jiménez, 2008) y el propio comportamiento social. Esto es especialmente relevante para la investigación en economía aplicada. La reciente concesión del nobel de Economía a Richard Thaler confirma que merece la pena considerar los elementos sociales y conductuales en el análisis de la economía y de las decisiones de la gente. Estos vienen determinados por el contexto social, por las expectativas compartidas por individuos y organizaciones dentro de sus universos simbólicos que no tienen por qué encajar con las propuestas de una acción racional establecida desde criterios supuestamente racionales apriorísticos. La idea de construir procesos económicos e instituciones donde la ciudadanía pueda decidir mejor y en libertad es una de los retos que se puede contrastar empíricamente, (Thaler y Benartzi, 2004).

Si desde una perspectiva microsociológica las emociones son un asunto clave que interviene en los rituales de interacción (Collins, 2013), la energía emocional de la que habla Collins también cobra un papel esencial en la economía aplicada. Esto se produce y se construye dentro de sistemas sociales donde la complejidad se incrementa en la medida que la digitalización avanza. Estamos instalados en procesos de anticipación. Unos son conscientes y accesibles, van desde la previsión y compra de planes de pensiones hasta la planificación de las vacaciones. Otros invisibles gestionados por servidores sirena (Lanier, 2014) que procesan millones de datos, big data, para establecer correlaciones eficaces de anticipación de comportamientos. Dos opciones que discurren entre la libertad y el control, en el amplio mundo de lo social

En lo futuro se interviene desde el presente. Hoy estamos construyendo el siguiente paso. Pensamos en mañana, donde lo futuro se anticipa y se construye. No sólo consiste en describir lo que puede pasar, no es suficiente con pensar escenarios posibles, también cabe intervenir para trazar rumbos, para elegir a dónde se quiere ir. El futuro es resultado de la voluntad, mejor dicho, de la suma de voluntades. Esto, precisamente, es una de las piedras angulares de lo social. Los códigos que nos permiten entender la vida cotidiana no sólo se producen por azar. Se construyen. Por eso es posible tomar decisiones, trazar rutas y arriesgarse a pensar. Aquello de sapere aude, vuelve a ser necesario. Quizá más que nunca. 


\section{REFERENCIAS BIBLIOGRÁFICAS}

AMOZURRUTIA, JA. (2012). Complejidad y sistemas sociales: un modelo adaptativo para la investigación interdisciplinaria. México. UNAM. Disponible en: http://computo.ceiich.unam.mx/webceiich/docs/libro/Complejidad_y_Ciencias_Social es.pdf [Último acceso: 11/10/2017]

ARAMBURU, L. MARCUELLO-SERVÓS, Ch. (forthcoming 2017). "Digital generation, emotions and social movements: A conceptual framework". En Manuel Lisboa (ed.) (2017): Complexity and Social Actions: Interaction and Multiple Systems. Cambridge Scholars Pub.

BOULDING, K. E. (1994). "Kenneth E. Boulding. De la química a la economía y más allá". En M. Szenberg, (ed.) (1994): Grandes economistas de hoy (pp. 79-95). Madrid: Debate.

BADKAR, M. (2017). "Euro hits highest level against the US dollar since Jan 2015 as Draghi speaks". Financial Times, August 25, 2017. Disponible en: https://www.ft.com/ content/a9be45f6-4c64-3464-9895-7fe82137068a [último acceso: 11/10/2017]

BERICAT, E. (2012). 'Emotions', Sociopedia.isa, DOI: 10.1177/205684601361, disponible en: http://www.sagepub.net/isa/resources/pdf/Emotions.pdf [Último acceso: 11/10/2017]

BOSTROM, N. (2001) "What is Transhumanism?", Disponible en, https://nickbostrom.com/ old/transhumanism.html [Último acceso: 11 de octubre de 2017]

COLLINS, R. (2013). Cadenas de rituales de interacción. Barcelona: Ed. Anthropos.

DRAGHI, M. (2017). "Sustaining openness in a dynamic global economy". Speech by Mario Draghi, President of the ECB, at the Economic Policy Symposium of the Federal Reserve Bank of Kansas City, Jackson Hole, 25 August 2017. Disponible en: https://www.ecb.europa.eu/press/key/date/2017/html/ecb.sp170825.en.html [Último acceso:11/10/2017]

DRUCKER, P. (1993). La sociedad post-capitalista. Barcelona: Ed. Apostrofe.

GALBRAITH, J. K. (2008). [1954] El crash de 1929. Barcelona: Ariel.

GARCÍA LIZANA, A. \& MORENO JIMÉNEZ, J.M. (2008). "Economía y Democracia en la Sociedad del Conocimiento". Estudios de Economía Aplicada 26(2), pp. 181-212.

POLANY, K. (1989). La gran transformación. Crítica del liberalismo económico. Madrid: Ed. La Piqueta-Endymion.

GRANOVETTER, M. (1985). "Economic Action and Social Structure: The Problem of Embeddedness". American Journal of Sociology, vol. 91, Issue 3 (Nov.1985), pp.481-510.

GÓMEZ FONSECA, M.A. (2004). "Reflexiones sobre el concepto de embeddedness". Polis: Investigación y Análisis Sociopolítico y Psicosocial, vol. 2, núm. 4, $2^{\circ}$ Semestre, 2004, pp.145-164.

HAN, B.-Ch. (2012). La sociedad del cansancio. Barcelona: Herder.

LANIER, J. (2014). ¿Quién controla el futuro? Madrid: Debate

MARCUELLO-SERVÓS, Ch. (2006). "La pregunta de Dorine". En MARCUELLOSERVÓS, Ch. (2006): Sociocibernética: Lineamientos de un paradigma. (pp. 5-22). Zaragoza. IFC. 
MARCUELLO-SERVÓS, Ch. (2010). "Digital Generations, Soft Societies?". Paper presented at Session: Understanding cyberspace and the Internet: Sociocybernetics on the Move Part 1. RC51, XVII ISA World Congress Of Sociology Gothenburg, Sweden.

MORENO-JIMÉNEZ, J.M. (2006): "E-cognocracia: Nueva Sociedad, Nueva Democracia" Estudios de Economía Aplicada, 24(1-2), pp. 559-581.

SCHEFF TJ (1990). Microsociology: Discourse, Emotion and Social Structure. Chicago. The University of Chicago Press.

SOTELO, I. (2010). El estado social: antecedentes, origen, desarrollo y declive. Madrid: Ed. Trotta.

THALER R H. and BENARTZI, S. (2004). "Save More Tomorrow: Using Behavioral Economics to Increase Employee Saving". Journal of Political Economy, 2004, vol.112, n.1. Disponible en: http://faculty.chicagobooth.edu/richard.thaler/research/ pdf/smartjpe.pdf [Último acceso: 11/10/2017]

VEEN, W. \& VRAKKING, B. (2006). Homo Zappiens. Growing up in a digital age. London. Network Continuum Education. 
